\title{
Experimental Validation of 2D Uncertainty Quantification for Digital Image Correlation
}

\author{
P.L. Reu ${ }^{1, \mathrm{a}}$ \\ ${ }^{1}$ Sandia National Laboratory, Albuquerque, NM, USA
}

\section{Introduction}

Because digital image correlation (DIC) has become such an important and standard tool in the toolbox of experimental mechanicists, a complete uncertainty quantification of the method is needed. It should be remembered that each DIC setup and series of images will have a unique uncertainty based on the calibration quality and the image and speckle quality of the analyzed images. Any pretest work done with a calibrated DIC stereo-rig to quantify the errors using known shapes and translations, while useful, do not necessarily reveal the uncertainty of a later test. This is particularly true with high-speed applications where actual test images are often less than ideal. Work has previously been completed on the mathematical underpinnings of DIC uncertainty quantification and is already published [1], this paper will present corresponding experimental work used to check the validity of the uncertainty equations. Sandia is a multiprogram laboratory operated by Sandia Corporation, a Lockheed Martin Company, for the United States Department of Energy's National Nuclear Security Administration under contract No. DE-AC0494AL85000.

\subsection{Experimental setup}

For comparison with the uncertainty quantification (UQ) equations a series of sub-pixel shifted images are required. Furthermore, these images should be experimentally obtained rather than numerically shifted in order to remove any questions of the integrity of the numerical shifting method. Ten sub-pixel shifted images were created using a single 14-Megapixel image taken with a Prosilica GE4900 CCD camera. The original image was $4872 \times 3248$ pixels; this was reduced via a decimation, which took every $10^{\text {th }}$ row and column and then writing it to a smaller image that was $487 \times 324$ pixels. By changing the start row or column chosen for the decimation, an exact virtual $1 / 10^{\text {th }}$ pixel shift was able to be generated. To ensure appropriate speckle size after decimation, the speckles were created to be approximately 50 pixels on the full-resolution image. This yielded 5pixel speckles after decimation. This speckle size was chosen to be large enough that low-fill factor aliasing should not be a problem. Figure 1 outlines the procedure and shows a set of example images.

This method of sub-pixel shifting has the great advantage of creating "perfectly" shifted images and simplifies the UQ analysis in that the noise of the measured displacement does not need to be taken into account. To create a valid data set for analysis and to avoid self-correlation, it was important to use two independent full-resolution images to create the decimated images. If only one image is used, the zero and 1-pixel shifted images are exactly the same and are self-correlated making the measured correlation errors incorrect. Because of this, a different image (although decimated via the same method) was used for the reference image. Two possible drawbacks result from this method:

This is an Open Access article distributed under the terms of the Creative Commons Attribution-Noncommercial License 3.0, which permits unrestricted use, distribution, and reproduction in any noncommercial medium, provided the original work is properly cited. 
the first relates to the possible aliasing of the speckle field. This is caused by the low fill factor in the decimated images created by taking every $10^{\text {th }}$ row/column. This has been avoided by creating speckles that are large enough after decimation (5 pixels) that they are not near the Nyquist frequency. The other drawback is that only perfectly horizontal or vertical shifting can be created.
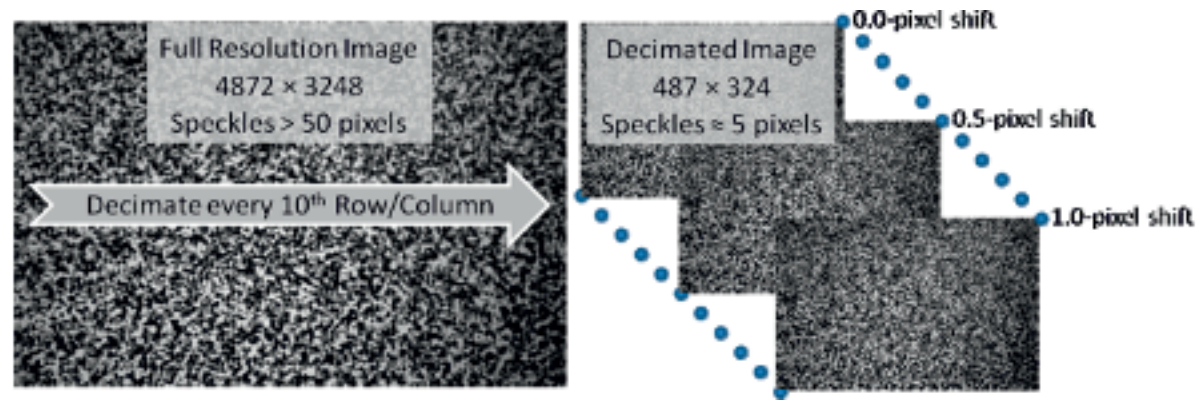

Fig. 1. Illustration of images created showing the original image and 3 of the 10 decimated images.

\section{Uncertainty quantification comparisons}

Uncertainty quantification equations will be presented which will be used to calculate the bias and variance errors for the decimated images. The results of the equations will then be plotted versus the $2 \mathrm{D}$ correlation results obtained from Vic2D. As the uncertainty depends on both the contrast of the images and the noise, both of these will be varied in a series of tests to obtain a range of uncertainties. Figure 2 shows a comparison of a "bad" image, with low contrast and lots of noise. The curve shows the typical sinusoidal bias error with error bars for the standard deviation of the calculated sub-pixel displacement. The straight line plotted is the UQ software results showing that the noise bias is dominant. The paper will present a thorough comparison of the experimental results and the UQ calculations.

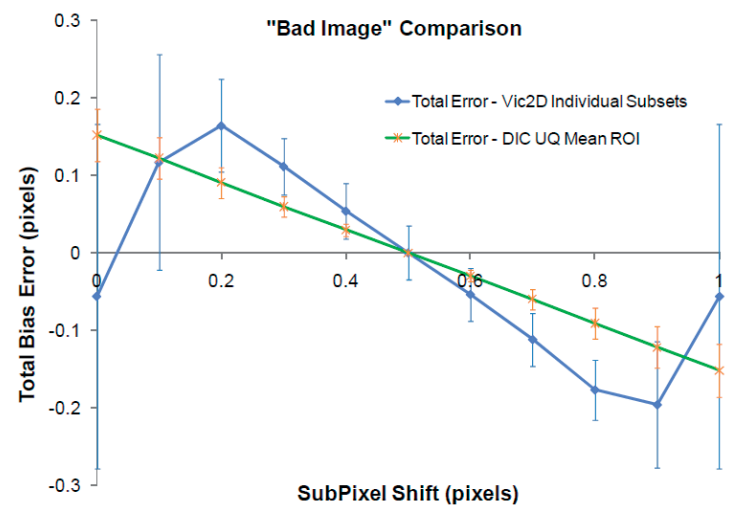

Fig. 2. Preliminary bias error comparison between UQ calculations and correlation errors.

\section{References}

1. Y.Q. Wang, M.A. Sutton, H.A. Bruck and H.W. Schreier, Strain 45, 2 (2009) 